\title{
VIENTOS: A FEASIBILITY STUDY OF INNOVATIVE PUPIL SYSTEMS FOR THE NEW GENERATION OF INSTRUMENTS IN THE LARGE TELESCOPES
}

\author{
García-Vargas, M.L. ${ }^{1}$, Pérez-Calpena, A. ${ }^{1}$, Gallego, J. ${ }^{2}$, Gil de Paz, A. ${ }^{2}$, Sánchez-Blanco. E. ${ }^{\text {, }}$ \\ Martínez-Delgado, I. ${ }^{1}$, Maldonado-Medina, M. ${ }^{1}$, Zamorano, J. ${ }^{2}$ \\ ${ }^{1}$ FRACTAL SLNE (Madrid, Spain), ${ }^{2}$ UCM (Madrid, Spain);
}

\begin{abstract}
The goal of VIENTOS project is to analyze pupil innovative systems that could be used in the new generation of instruments for the large telescopes. This study tries to identify the current scientific needs, to understand why some of them have not been fulfilled yet (due to pre-conceived technical ideas or to managerial reasons) and to propose optomechanical solutions for these pupil elements that could produce a qualitative leap in the performance of the instruments to operate in the large telescopes. VIENTOS is currently on-going as a collaborative project between FRACTAL and the University Complutense of Madrid (UCM) and is being partially funded by a CDTI grant under the program Industry for Science. CDTI is the Development and Industrial Transfer Center from the Minister of Science and Innovation (Spain).

Among the different innovative systems that we have carried out, our team has explored potential solutions for narrow band Imaging with tunable filters in the near-IR and a novel pupil system called sliced-pupil grating, a device designed for increasing the spectral resolution in astronomical spectrographs, without changing the geometry of the main optics. Nanotechnology customized filters to be applicable to astronomical systems are under study.
\end{abstract}

Keywords: Pupil elements, tunable filters, large telescopes, gratings, VPHs, spectral resolution, sliced-pupil grating.

\section{INTRODUCTION}

The VIENTOS ${ }^{1}$ project was presented in the announcement of opportunity named Industria de la Ciencia (Industry for Science) that was raised by the CDTI in May 2010 and, after a successful evaluation, it was approved in October 2010. The project spans from the July 2010 to October 2012. It is a collaborative project developed between FRACTAL SLNE and the Universidad Complutense de Madrid (UCM) an co-funded by these two institutions in addition to the CDTI grant.

The VIENTOS project proposes to conduct a feasibility study to analyze pupil innovative systems that could be used in the new generation of instruments for the large telescopes. This study involves to identify the current scientific needs, to understand why some of these needs have not been yet fulfilled (due to technical or managerial reasons or to preconceived ideas) and to propose opto-mechanical solutions for these pupil elements that could produce a qualitative leap in the performance of the instruments that are going to be used in the large telescopes.

The aim of this study is twofold: firstly to increase the FRACTAL capabilities in order to raise the visibility of FRACTAL in this international area and to fill a niche market not yet covered: the design of opto-mechanical systems applied to astronomical instrumentation; and, also, to improve the position of the Universidad Complutense de Madrid in the international instrument consortia, in which they are already well known for the development of instrumentation data reduction software, but in which the development of high-technology opto-mechanical components provides an

\footnotetext{
${ }^{1}$ VIENTOS project is being co-funded by CDTI, FRACTAL and UCM. This work has been done also in the framework of the Moncloa Campus of International Excellence initiative (UCM-UPM)
}

Ground-based and Airborne Instrumentation for Astronomy IV, edited by lan S. McLean, Suzanne K. Ramsay, Hideki Takami, Proc. of SPIE Vol. 8446, 84467T · — 2012 SPIE · CCC code: 0277-786X/12/\$18 · doi: 10.1117/12.926062 
additional prestige and value as this particular field is highly demanded. Some of the activities have lead to the design of customized instruments already planned for the GTC, like a tunable filter for CIRCE and a sliced pupil for MEGARA ${ }^{1}$, being both approved instruments for the GTC 10-m telescope, currently in operation in the Observatory of Roque de los Muchachos, a the island of La Palma (Canary Islands, Spain).

This paper provides an overview of some activities already performed in the VIENTOS project: (a) the increasing of spectral resolution by using a novel pupil system called sliced-pupil grating, (b) the feasibility analysis for achieving narrow band imaging with tunable filters, (c) the market analysis about using VPHs in Infrared, with strong emphasis in the E-ELT instruments and (d) the activities performed to improve the capabilities of the LICA-UCM laboratory set-up for testing large-format pupil optical components. Finally, just to mention that VIENTOS project is still on-going and, during this last year, we plan to study the feasibility of producing nanotechnology customized filters, we could eventually open a new market to build cheaper and high performance filters whose transmission and pass-band are defined by the deposition of nano-particles, and by controlling the number, size, nature and distribution of these particles in different kind of substrates.

\section{SLICED-PUPIL GRATING DESIGN AND PROTOTYPE OUTCOMES}

The sliced-pupil grating is an innovative pupil device that can allow multiplying by three or four the spectral resolution of current spectrographs without changing the geometry of the instrument.

A fixed and straight geometry is widely used in astronomical spectrographs to keep instrument compactness. Due to this reason, the angle of incidence on the grating is very limited and the attainable resolving power, $\mathrm{R}$, is usually lower than 5000. The problem becomes harder for large pupil with a strong difference in incident angles on pupil. In addition, there were few spectrographs providing mid-resolution $(\mathrm{R} \sim 10000-20000)$ in professional observatories and none of them can simultaneously provide a lower $(<5000)$ or a higher regime $(>50000)$ simultaneously. One of the reasons for that is the difficulty, in the optical design, of having different spectral resolutions regimens sharing the same geometry for the main optics (collimator-camera). This problem becomes even more relevant in large and giant telescopes, affecting from manufacturing problems in large pupil elements. The standard solutions, depending of the target spectral resolution, have been the use of echelle gratings, which implies to have a single or a much reduced number of observed objects, or the use of grism-type elements, by accommodating prisms at both sides of a transmission grating (VPH-type for throughput optimization) in order to increase the incidence angle on the grating and therefore the spectral resolution. However, the problem with this extended concept is that in these pupils, the prisms needed to get high angles for high resolution are too thick and complex, and the system becomes inefficient (in terms of transmission) and difficult to be assembled.

We have developed the opto-mechanical design of a novel spectroscopic element - the sliced pupil grating - that allows increasing the spectral resolution while keeping the angle between collimator and camera, and therefore the same geometry. The concept is based on "cutting" the pupil into different slices by placing a number of prisms at the two sites of a VPH grating. The independent beams are guided through a very precise opto-mechanical assembly to assure the recombination on the individual images on the detector within the available error budget to produce a single spectrum.

To probe the feasibility of the concept, we have designed, manufactured and tested a prototype unit able to produce high resolution $(\mathrm{R}, \lambda / \Delta \lambda \approx 10000)$ in the visible range (centered at $\mathrm{H} \alpha 6563 \AA$ ) in an already-built instrument (Elmer for the GTC 10-m telescope) originally designed for $\mathrm{R}=2500$ (for a 0.6 " wide slit) in Littrow configuration, and with straight in-line collimator-camera geometry $\left(0^{\circ}\right.$ incidence angle on pupil). This prototype has been built with three slices over an $89 \mathrm{~mm}$ pupil aperture. We have performed the components' individual tests as well as the system assembly at the Laboratory for Advanced Scientific Instrumentation, LICA, at the UCM. As a next step to use this concept in spectrographs for large and giant telescopes our group has designed a model of a 2-slice grating for the instrument MEGARA, a fiber-fed IFU and multi-object spectrograph for the GTC 10-m telescope. This will allow having resolutions of $\mathrm{R}=6000,11000$ and 18000 with the same geometry (FWHM), for a fiber-core width of $100 \mu \mathrm{m}$, and reaching up to $\mathrm{R}=25000$ for $70 \mu \mathrm{m}$ fiber core (see [R.1]. The higher spectral resolution configuration is obtained thanks to the sliced-pupil grating. MEGARA is lead by UCM (Spain), with the participation in the Consortium of INAOE (México), IAA (Spain) and UPM (Spain). MEGARA sliced-pupil gratings scientific requirements have been given by the MEGARA scientific team and the elements shall be designed by FRACTAL, and manufactured by Wasatch Photonics (VPHs holograms) and INAOE (prisms and windows). 
The following sections present the optical and opto-mechanical design of the 3-slices prototype (done for Elmer instrument as mentioned above) as well as the measured performed and results obtained from this prototype. A new 2slices design for MEGARA is being carried out during 2012, along the detailed design phase of MEGARA project.

\subsection{Optical design}

The optical concept is shown in Fig. 1 (left). The unit is composed of six prisms (three on each side) and the grating, consisting on a VPH hologram sandwiched between two flat windows. The VPH has 3400 lines $/ \mathrm{mm}(\mathrm{R}=10000$ at first order).

In order to obtain the high $\mathrm{AOI}$ on the grating for the required resolution, three prisms will slice the beam in three portions. A TIR on the upper faces of these three prisms will supply the required incidence angle on the grating. A gap among the three prisms at either side will be used to avoid tunnel transmission among the prisms. The prisms seat on the gel-coupled VPH window. After diffraction the beam will be redirected again using TIR on 3 new prisms to the camera.

The camera (Fig. 1, right) has four elements: two doublets and two individual lenses, with a focal length of $124 \mathrm{~mm}$. In the image different colors indicate different wavelengths. The material for the window and prisms is fused silica.

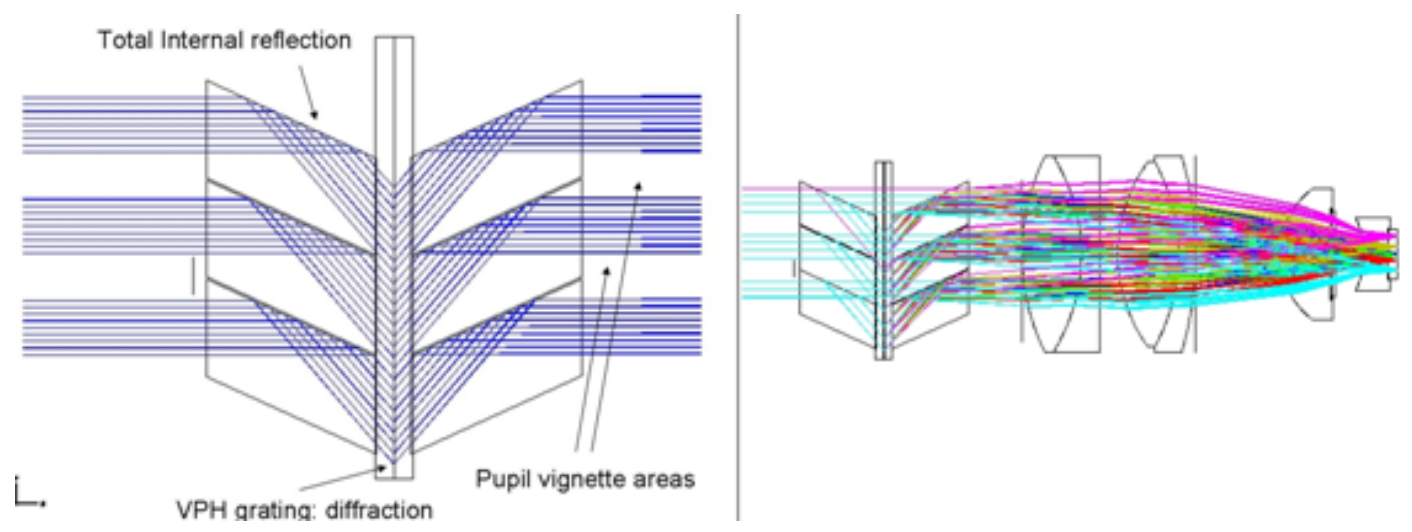

Fig. 1. Left: Sliced Pupil Grating concept. Right: layout of the slicing device with Elmer camera.

The unit is placed at the pupil $(89 \mathrm{~mm} \varnothing$ size) with a clear aperture of $105 \mathrm{~mm}$ that allows covering all unvignetted field at the edges. Nevertheless, as the pupil is sliced in three pieces to minimize assembly difficulties, some parts of the pupil's flux are lost (producing vignetting). In fact only $62 \%$ of the incoming pupil beam passes through the system. The unit is also de-centered $4 \mathrm{~mm}$ to accommodate the beam, going downwards after the TIR reflection the unit. Other designs, which have been optimized together with the main instrument, have allowed us to get transmission over $72 \%$.

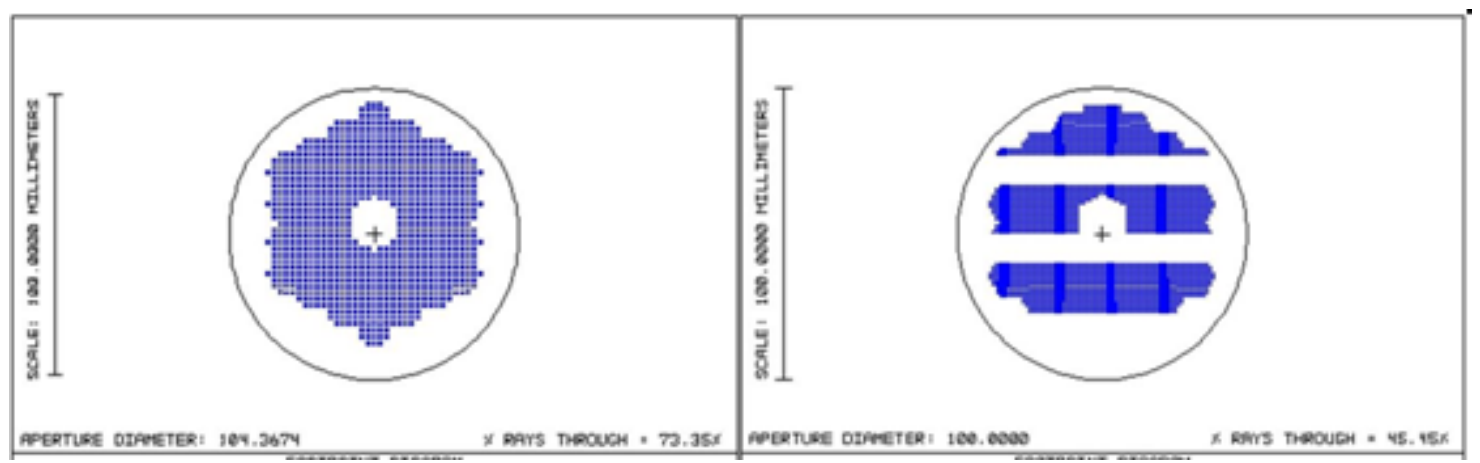

Fig. 2. On the left the nominal pupil of the central field at $656.3 \mathrm{~nm}$ before crossing the dispersive element. On the right the vignetted areas are shown. Only $62 \%$ of the pupil area is expected to pass through the aperture. 
Difficulties arise when we consider that after the beam slicing, we have to recombine the three different paths. In fact the perfect scenario would be to recover the path without delays among the beams. We do not intend to recover this coherence as the beam through the atmosphere is already corrugated. We can estimate the wavefront rms (equation 1) as:

$$
\text { FWHM (rad) }=0.98 \times\left(\frac{\lambda}{R_{0}(\lambda)}\right)
$$

, where $\mathrm{R}_{0}$ (Fried parameter) is $22 \mathrm{~cm}, \lambda=656.3 \mathrm{~nm}$ and FWHM $=0.6 "$. The wavefront $\mathrm{rms}$ is given in equation 2 .

$$
\sigma^{2}=1.023\left(\frac{d}{R_{0}}\right)^{\frac{5}{3}}
$$

, for this seeing and a $10.4 \mathrm{~m}$ telescope. The result for $\sigma=25$ radians, is about $4 \lambda \mathrm{rms}$, or $12 \lambda \mathrm{P}-\mathrm{V}$. Our strategy is based on common image stacking not far from the atmospheric maximum departure. As the unit will work above the diffraction limit, we do not expect large effects due to the beams interference (only few percent).

A complete description of the optical design and analyses can be seen in Sánchez-Blanco et al. $2011^{2}$

\subsection{Opto-mechanical design}

In order to place the optical elements in their exact position within the instrument optical path, they must be inserted on a mount that interfaces with the Elmer instrument prisms wheel.

This mount allows placing the different optical elements in its exact position relative to each other as well as locating the whole grating with precision onto the optical path, both defined by the optical design. The mount has been designed to absorb the differential thermal dilatations of the optics with respect to the aluminum mount, maintaining its performance, and preventing surface stresses that may affect the transmission.

The optical design presented in the previous section is quite symmetrical, with a central flat window and three prisms on either side. The hologram is embedded in the central element, which in fact is a sandwiched window. The mount must leave an optical aperture of $\varnothing 105 \mathrm{~mm}$, with its centre on the optical axis of the instrument. It must also comply with the envelope provided on the prism wheel, and keep its maximum size between limits defined by the distance between the camera and the filters wheel. It is desirable that the mount stays as near as possible to the camera to assure the best aperture without vignetting and prevent stray light. Fig. 3 shows a 3D model of the mount and the real element with the dummies of the prisms during the tests. More details can also be seen in Sánchez-Blanco etal. $2011^{2}$.
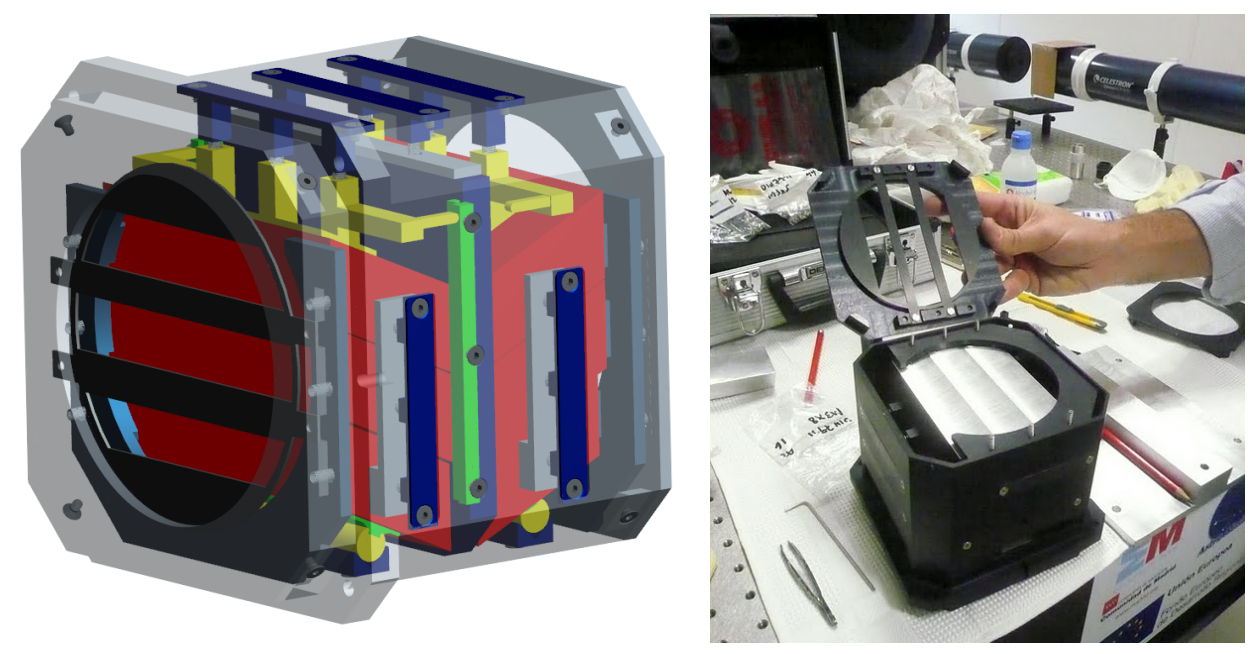

Fig. 3. Left, 3D view of the overall grating design. Right, real element with aluminum dummy prisms during tests 


\subsection{Tests and outcomes}

This section summarizes first the test performed at LICA-UCM to the optical components (prisms and VPHs) before integrating them in the assembly in order to verify that fulfill the requirements; then, the mounting process followed to integrate whole assembly; and, finally, the measurement taken in the sliced pupil grating and the conclusions reached.

\section{Prism tests}

The prisms used in the prototype were manufactured by SESO. Besides the technical and design requirements, one of the tightest specification was to obtain and cut the prisms used in the spectrometer, from the same block. Two blocks were used, obtaining three prisms from every one. This should let the prisms to be more similar and homogeneous among them (surfaces, angles, etc.), and in case no defect be produced in the manufacturing process, the small homogeneities in the prisms due to the own glass crystallization properties should be similar among them. We measured the parallelism of the surfaces, the surface quality, the angles between the prism surfaces and the equality of the angles (see Fig. 4).

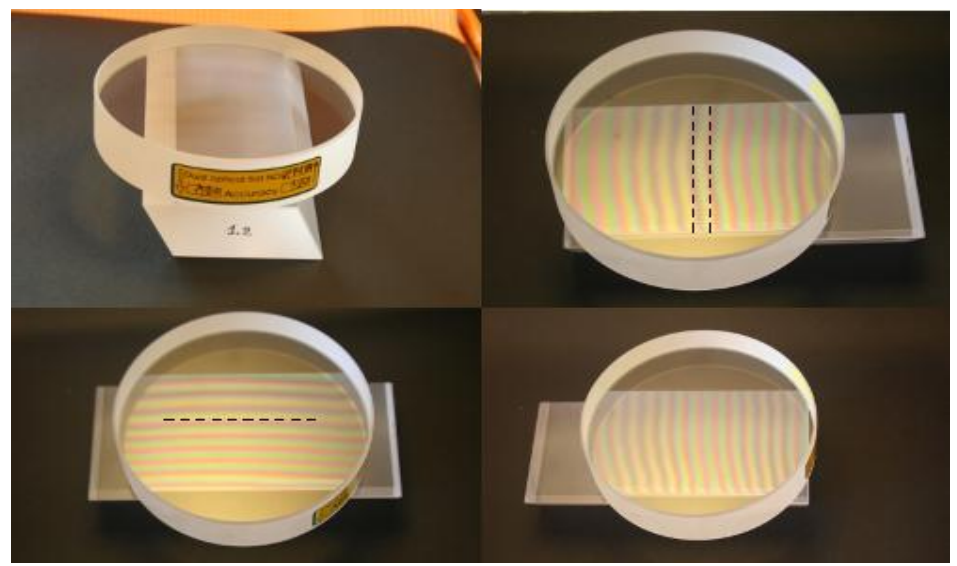

Fig. 4. Measurement of the surface flatness over the prism's surfaces.

All the tests performed at both, SESO and LICA confirmed that all prims parameters were within the required tolerances.

\section{VPH tests}

The VPHs were manufactured by Wasatch Photonics, who sent us several gratings fulfilling the same specifications. We performed the measurements to verify the specification fulfillment and to assess which VPH provides the best quality and use this unit for the assembly. The tests performed include dimensional verification, windows surface quality, number of lines/mm per grating and image quality (PSF Star tests). All VPHs fulfilled specifications.
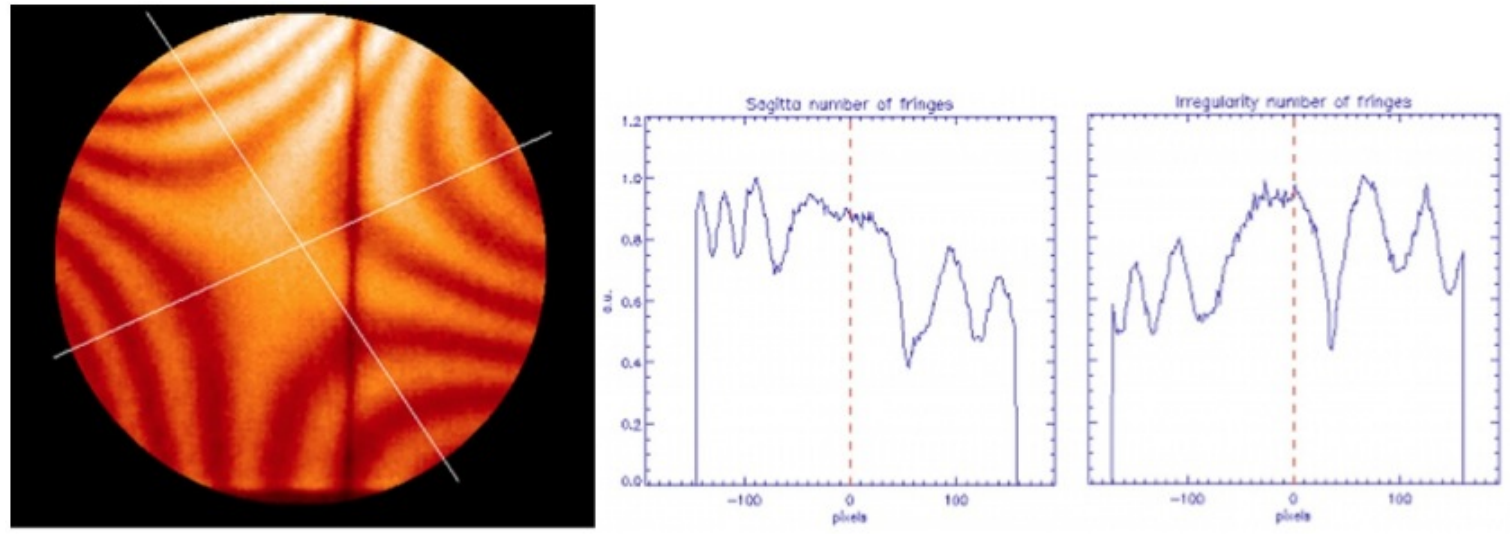

Fig. 5. Fringe map of the surface of one of the VPHs. The circle corresponds to the pupil size. The two white solid lines over-plotted show from where have been taken the bottom profiles (Sab) and (Iab) profiles respectively. 


\section{Mounting sequence}

The mounting process begins placing the grating hologram on the reference surfaces of the inner side of the main body of the mechanical mount, being the mount in vertical position. Once the VPH is fixed to the system, we begin with which is probably the most delicate step: to face the prisms to the VPH and fitting them together.

For this step it was used an epoxy NOA 61 due to the following reasons: (a) the refractive index of this material is closed to the refractive index of the fused silica (the material from the prisms and the VPH are made), which is very important for not leading to a great deviation of the light beam in the interface between the prisms and the VPH; (b) it is a photopolymer that cures when it is exposed to UV light needing several minutes for curing (between 5 and 10 min with 100 watt mercury lamp), what give us a time operation margin in order to adapt the prism and/or take out the prism if the polymer hasn't spread homogeneously over the surface, or if it has overflowed the contact surface, etc. and (c) the adhesive is designed to give the best possible optical bond to glass surfaces, specially treating with lenses, prisms, etc.

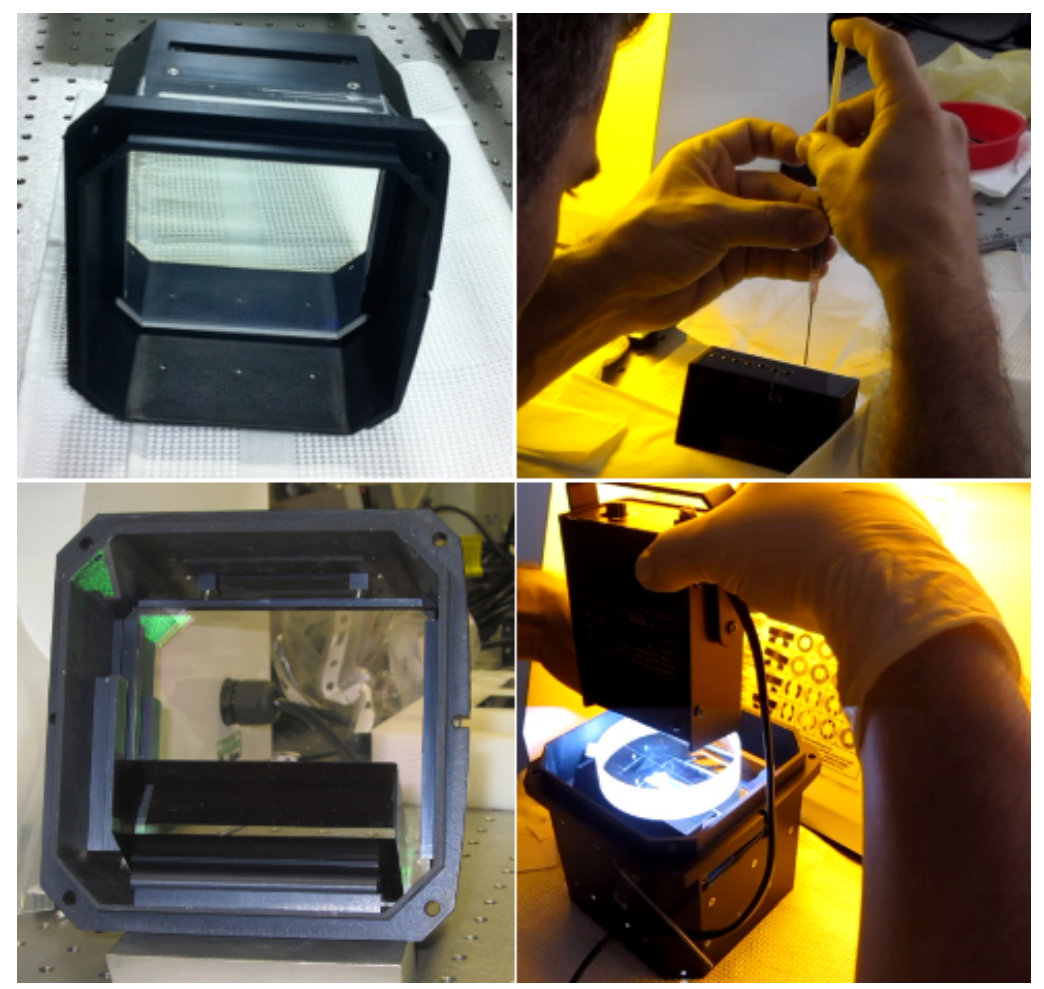

Fig.6. Top left.- the VPH once this one has been fixed to the inner side of the mount. Top right.- Dropping the epoxy on to the surface that will make contact with the VPH. Bottom left.- View of the first prism nominal position over the pivot device and making contact with the VPH. Bottom right.- Curing the polymer with the ultraviolet lamp. The flat reference surface is placed over the three prisms for controlling the adhesive process and correcting any deviation tilt before the cure is irreversible.

The polymer must be put in the prism over the surface of interest with precision and equally spaced in order to help the adhesive to spread itself homogeneously when the contact between surfaces has been done. Once the prism is coupled to the surface and the polymer has extended homogeneously over the contact surface is time for photo curing the adhesive with an ultraviolet lamp. Because the cure of the polymer is irreversible, the optical quality of the surfaces that are in contact must be checked. In Fig.7 (top left) we can see the final result of the adhesive process. The figure shows that the central prism is coupled perfectly while the other two shows a small tilt in the spatial direction, and do not affect the spectral direction (parallel to the dark line). So the final conclusion is the good coupling of the three prisms.

Once the optical coupling of the system is checked, the time to insert the stainless steel sticks with the Araldite epoxy, as it is shown in Fig.7 (top right). Later, the system can be placed horizontally and we can continuous mounting the baffle that stop the light that goes directly to the air interface between each prism. All this process must be repeated for the other side. 


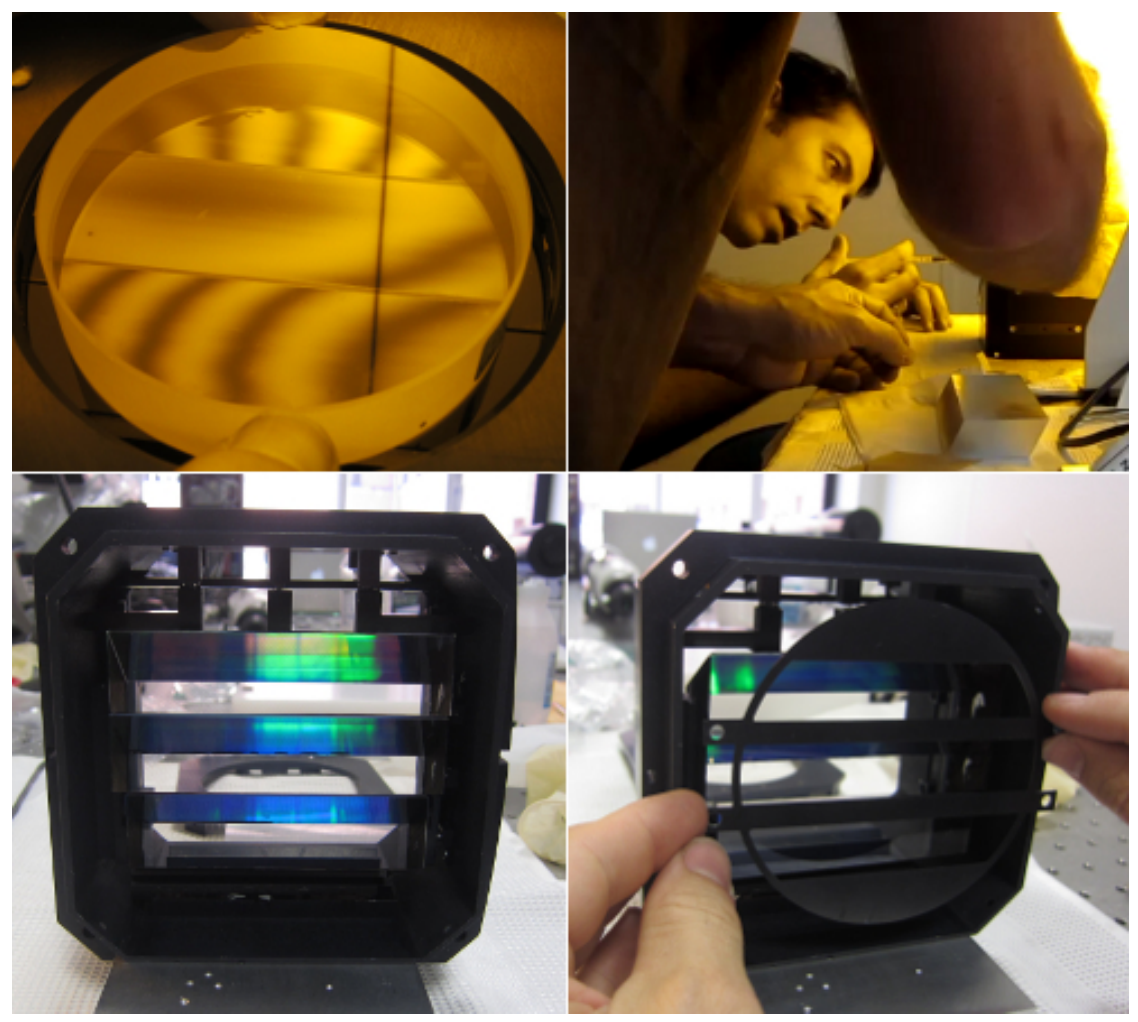

Fig.7. Top left.- Testing the final quality of the surfaces fixed to the VPH. Top right.- Inserting the epoxy where the sticks are placed. Bottom left.- The system in its nominal position once the adhesive is completely cured. Bottom right.- mounting the baffle mask.

\section{Sliced pupil grating assembly tests}

Once the assembly process has finished it is time for testing and taking the first results from the disperser.

The main test is to check that the our prototype reaches as best as possible the last requirement, that is, the disperser is able to increase de nominal power resolution of ELMER.

For that we use two $900 \mathrm{~mm}$ of focal refractive telescopes facing each other and previously collimated, one of them used as a collimator and the other one as a camera, with the disperser placed in between.

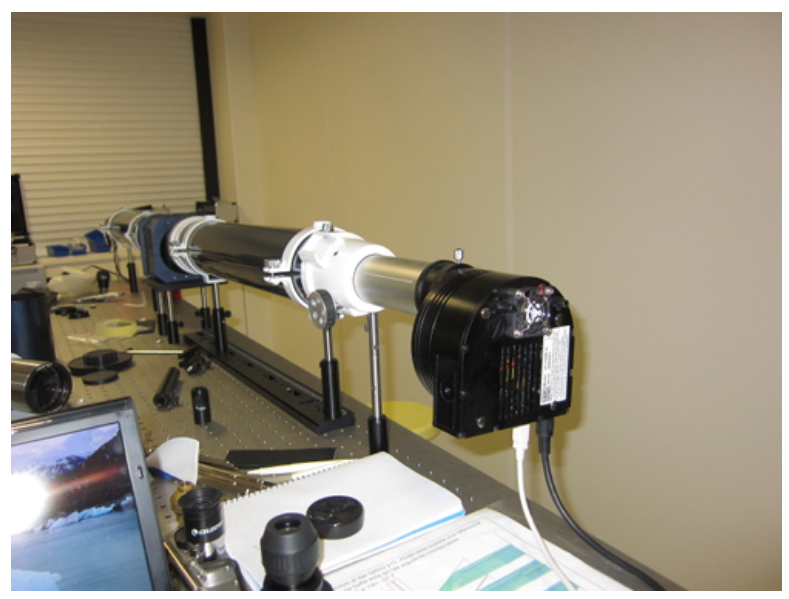

Fig. 8. Optical system compound by the two telescopes used for obtaining a measure of the resolution. 
As the focal lengths of collimator and the camera are the same, our configuration is an anamorphic system. The focal plane of the collimator is provided with a pinhole of $25 \mu \mathrm{m}$ and/or a $25 \mu \mathrm{m}$ slit, and the focal plane of the camera is provided with a ST-7 CCD device with a FoV of 764 x 509 pixels and a pixel size of $9 \mu \mathrm{m}$. The aim of this optical configuration is to obtain a precise image of the $\mathrm{H} \alpha$ light passing through the system and to measure the final power resolution derived.

That is, the principle of our disperser is based on the technique of slicing the pupil; if we use the light coming from a point source, when its beam of light pass through the disperser this is split into three beams (having three points, one for every prisms) that should be refocus into one point by the camera. That should be the ideal system.

If we have a look to Fig. 9, we can see that the final reconstruction of the exit beam has not been completely successful. In the left side of the figure it is shown the disperser system with a numbering label inserted over every prism that match those found in the right side of the picture, where it is shown the three point sources produced by the light that comes from every prism. This deviation is completely due to assembly process errors being the most important that one related with the prisms accomplishment into the VPH surface. At this level if the polymer layer between the contact surfaces is denser in one point than in the rest of the surface, a bit tilt is generated between each prism and the VPH, producing a deviation of the exit beam, and by the way, there is a degradation of the final image.

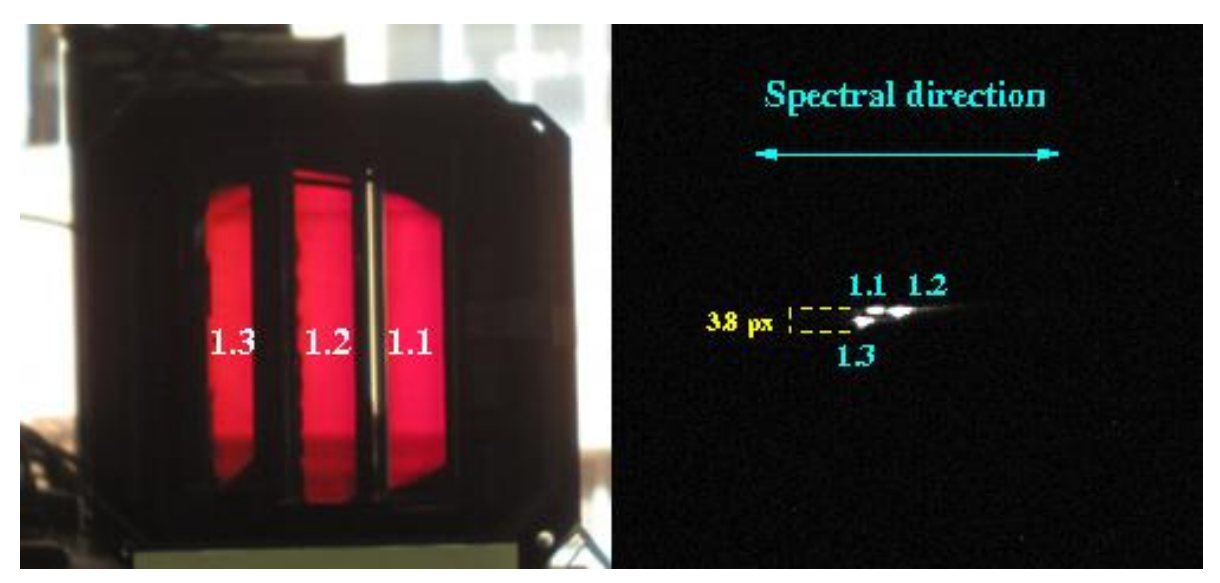

Fig.9. Left side.- the disperser system with label over-plotted numbering the position of the prisms; right side.- image of the three Ha point sources and their correspondences with the prism that produces image. Deviation equivalent to ELMER's pixels is given.

The image produce by all the optical system could be obtained as:

$$
P^{\prime}=\frac{f 2}{f 1} \mathrm{P}
$$

As the collimator and the camera have the same focal distances ( $\mathrm{fl}=\mathrm{f} 2=900 \mathrm{~mm}$ ) we have that $\mathrm{P}^{\prime}=\mathrm{P}=25 \mu \mathrm{m}$, and therefore in the ST-7 CCD has a size of 2.78 pixels. The three points are located at the following position:

\begin{tabular}{|c|c|c|}
\hline Point & $\mathrm{X}$ & $\mathrm{Y}$ \\
\hline 1.1 & 267 & 306 \\
\hline 1.2 & 286 & 306 \\
\hline 1.3 & 257 & 315 \\
\hline
\end{tabular}

Table 1. Pixels position of the three point sources imaged at the CCD 
So the maximum distance found in the spectral direction (that is in the $\mathrm{x}$ direction) between the most distant sources is 29 pixels. If we de-convolution to this distance the pinhole image size we have:

$$
\sqrt{29^{2}-2.78^{2}}=28.866 \text { pixels }=0.25979 \mathrm{~mm}
$$

So this is the size of the point source in the ST-7 camera, and therefore the size of a similar point source seen from ELMER should be obtained by means of the following trigonometrical relation:

$$
\theta 1=\frac{0.25979 \mathrm{~mm}}{900 \mathrm{~mm}}=\theta 2=\frac{\text { Xsize }}{\text { ELMER' sfocal }}
$$

The focal distance of ELMER is $\mathrm{f}=123 \mathrm{~mm}$, so the point source observed with ELMER would have a size of $\sim 35.50 \mu \mathrm{m}$ $=2.37$ pixels (being the pixel size of ELMER of $15 \mu \mathrm{m}$ ).

The resolution element of ELMER considered in our optical design, in order to reach a power resolution $\mathrm{R}=10000$ is 3 pixels. So the degradation obtained in the resolution element is given by the following convolution:

$$
\sqrt{3^{2}+2.37^{2}}=3.82 \text { pixels }
$$

Therefore, if we had $\mathrm{R}=10000$ for 3 pixels in our theoretical system, the final $\mathrm{R}$ reached in the prototype is 7850 . So with this prototype the nominal resolution of ELMER $(R=2500)$ has been increased by a factor of 3 .

As conclusion, we consider that the prototype was quite successfully to prove the feasibility of the concept. The sliced pupil grating has been able to increase the resolution of the instrument in more than 3 times (from $R=2500$ to $\approx 8000$ ), although the upper limit of the resolution noted at the requirements $(\mathrm{R}=10000)$ was not reached. The experience gained with the prototype shall allow us to improve the mounting process that should be done for further units registering the stacking while gluing the prism to verify that the three beams are going into a common point at the focal plane.

If required, we could even improve the prototype resolution to reach $\mathrm{R}=10000$ applying a correction in the exit paths of the light and drive the three beams into a common point at the focal plane. The idea would be to apply a correction mask at the exit pupil of the disperser, based on three small prisms that re-drive the ray paths to a unique and a single point into the focal plane. With this mask the final resolution will reach the value of $\mathrm{R}=10000$.

\section{TUNABLE FILTER FEASIBILITY STUDY}

The priority of this piece of work was to study the implementation of tunable filters in the near-IR up to K band, which has not been included in any instrument up to now. In particular, we study the possibility to include a cryogenic near-IR tunable filter (FISIR) for CIRCE, an instrument proposed by the University of Florida as visitor instrument for the GTC $10-\mathrm{m}$ telescope. FISIR is an initiative of Universidad Complutense de Madrid and University of Florida.

The FISIR requirements are that it shall be capable of scanning any wavelength in the near-IR range (from 1.0 to $2.5 \mu \mathrm{m}$ ) with a spectral resolution of about $\mathrm{R} \sim 750$ (set by the main scientific driver that is the program ALBA).

In this section we describe first the Fabry-Perot interferometer concept and tandem etalons performed for FISIR.

\section{Fabry-Perot concept}

Fabry Perot interferometer consists of a pair of identical transparent plates, having plane-parallel internal faces of reflectivity $\mathrm{R}$, separated by a uniform spacing $\mathrm{d}$. Peak transmission is attained over a series of orders $\mathrm{n}$ when

$$
2 \mu \mathrm{d} \cos \theta=\mathrm{n} \lambda \quad \mathrm{n}=0,1,2,3 \ldots,
$$

where $\mathrm{n}$ is the refractive index of the medium between the plates, and $\theta$ is the angle relative to the normal of the incident beam with wavelength $\lambda$.

The spectral distance between two adjacent orders $n$ and $n+1$ is called the free spectral range (FSR), and is given by

$$
\Delta \lambda \operatorname{FSR}=\lambda / \mathrm{n}
$$



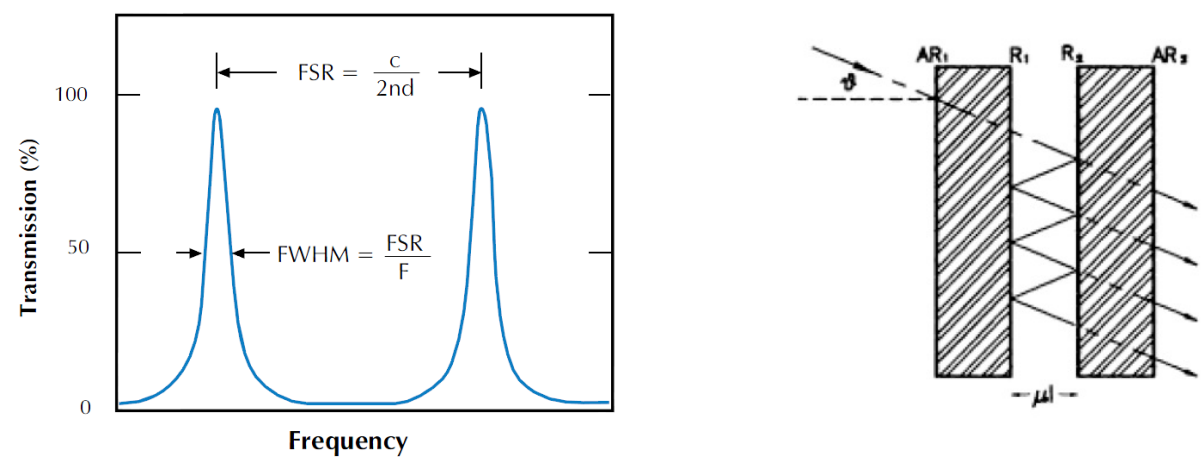

Fig. 10. The transmission of an etalon (Left). A Fabry - Perot etalon comprising two glass plates with highly reflective interior surfaces, R1 and R2, and anti-reflective exterior coatings, AR1 and AR2 (Right).

For a 'perfect' Fabry - Perot etalon, the full width at half-maximum (FWHM) of each order is

$$
\Delta \lambda \mathrm{FWHM}=\lambda(1-\mathrm{R}) /\left(\mathrm{n} \pi \mathrm{R}^{1} / 2\right)
$$

Thus the resolving power $\mathrm{R}$ can be described as

$$
\mathrm{R}=\lambda / \Delta \lambda \mathrm{FWHM}=\left(\mathrm{n} \pi \mathrm{R}^{1} / 2\right) /(1-\mathrm{R})=\mathrm{n} \text { FR }
$$

where FR is called the reflection finesse.

In practice, the true 'effective' finesse Fe of a Fabry Perot system is always less than the reflection finesse, due to surface defects in the coatings, departures from plate parallelism, and the use of a converging, rather than a parallel, incident beam. Replacing FR with Fe in equation (10) then allows the resolving power to be estimated in the general case.

Finesse is dominated by mirror losses. If mirrors were fully reflective, they wouldn't allow light in or out, and if they were a little reflective, the etalon wouldn't sustain an adequate amount of light power in it. The first mirror should be almost perfectly reflective (99.5\%) and the second mirror should be typically about $93 \%$ reflective.
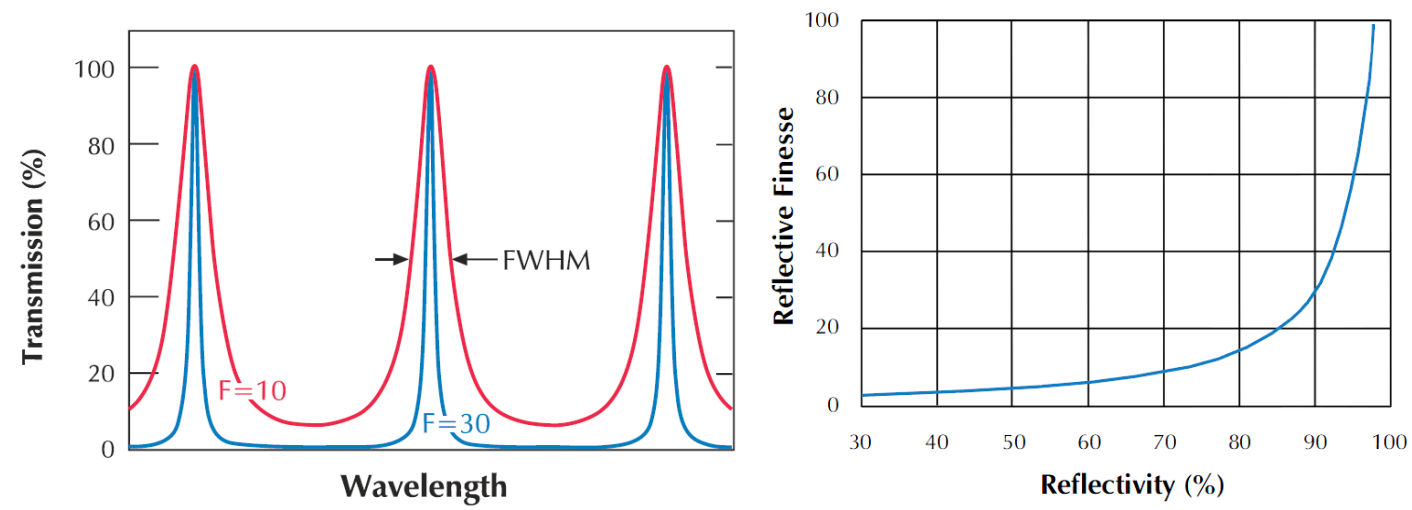

Fig. 11. Left side - The transmission of an etalon as a function of wavelength. A high-finesse etalon (blue line) shows sharper peaks and lower transmission minima than a low-finesse etalon (red). Right side - Relationship between reflectivity and reflective Finesse.

\section{Tandem etalon}

The Fabry-Perot interferometer can measure radiances over very small frequency bandwidths with a very significant throughput advantage over a grating spectrometer. However, the Fabry-Perot interferometer will only work if we can suppress the transmission side-lobes. This can be achieved using an interference filter with a bandpass narrower than the free spectral range of the etalon. This can also be achieved by using two etalons in tandem or in series. In other words, a first coarse etalon could be used to pre-filter the incoming radiation and suppress the near side-lobe transmission of the main etalon. Suppression of all main etalon side-lobes can be achieved with judicious choice of the first etalon gap and additional interference filter filtering. 
For ideal interferometers the overall transmission profile is simply the product of the transmission profiles of the two FPI. For real interferometers, however, it is important to take into account the effects of the surface defects. In actual use, the operation of two FPI in series requires a tuning process, in which the spacing of each interferometer is adjusted to have, at a desired wavelength, an optimum alignment between the transmission profiles of the two FPI resulting in a maximum overall integrated transmission.

\section{FISIR Optical analysis}

In order to evaluate the FISIR performance in CIRCE, the main optical parameters are reviewed in three positions; near the pupil, and near the two focal planes, FP1 and FP2. The current FOV is $3.36 \operatorname{arcmin} \times 3.36 \operatorname{arcmin}\left(4.75^{\prime}\right.$ in diameter).

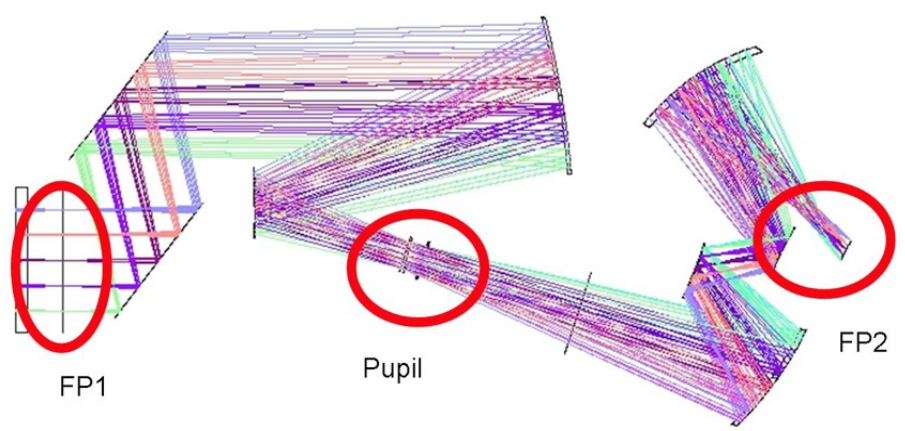

Fig. 12. Optical system compounds by the two telescopes used for obtaining a measure of the resolution.

The optimum case would be that all AOI are the same for the entire pupil (collimated beam) and for all the fields (telecentricity condition). This condition does not exist in the current design. The best position would be FP1, which contains the lower AOI angles, followed by the pupil position. FP2 is discarded because is the position where the beam contains the highest angles.

\section{Fabry-Perot etalon at pupil position}

At the pupil position each source arrives collimated, although different sources across the FOV arrive with different angles. The total size of the etalon aperture is minimized (from 70 to $90 \mathrm{~mm}$ ) while each field cross section is of the size of the pupil $(50 \mathrm{~mm})$. Thus the optical quality of the etalon surfaces has to be met in this aperture. The wavelength selection is provided by two consecutive etalons and an isolation bandpass filter. Each etalon is adjusted with a different cavity size.
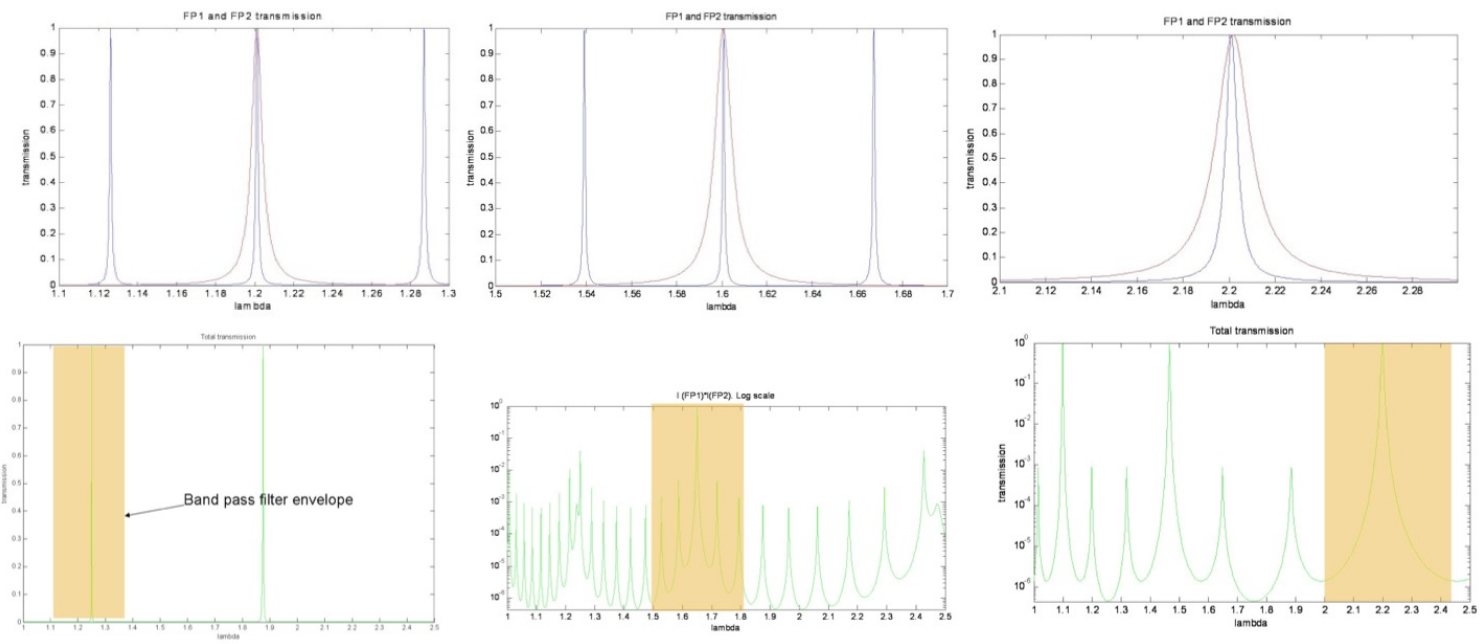

Fig. 13. Upper - Band-pass profile for both etalons $(1.25 \mu \mathrm{m}$, left; $1.60 \mu \mathrm{m}$, center; $2.2 \mu \mathrm{m}$, right). Bottom - Band-pass through all the spectral range in a log scale. Notice that a filter could be required in the $\mathrm{J}$ band (left), $\mathrm{H}$ band (center) and $\mathrm{K}$ band (right). 
Figure 13 shows the short spectral profile of several cavities centered at several wavelengths ( $\mathrm{J}$ Band at $1.25 \mu \mathrm{m}, \mathrm{H}$ Band at $1.60 \mu \mathrm{m}$ and $\mathrm{K}$ Band at $2.2 \mu \mathrm{m}$, all at $\mathrm{R}=700$ ). The $\mathrm{AOI}$ are always between $\pm 3^{\circ}$.

\section{Fabry-Perot etalon near the entrance focal plane}

The FP position near the focal plane has different characteristics compared to the same unit at the pupil position. The main advantages are that the rays for the different fields are basically parallel, thus a single exposure covers the whole FOV at the required wavelength and the used apertures (were the optical flatness is required is much smaller).

Otherwise, the disadvantages are that the total aperture is much larger, thus the mechanical assembly could be more complex and will be more expensive; and, also, as the total aperture is bigger, the cavity coatings will be more expensive as well as the blanks. We must analyze if there is FWHM degradation in the band-pass due to the different angles of the F17 cone for each field and if the ultimate Finesse is also limited due to the change of the used aperture.

The AOI on the FP at the focal plane is driven by the cone angle (F17) as seen in Fig. 14 (Left). The marginal ray is coming at $1.65^{\circ}$ maximum AOI from the normal surface. Thus different pupil sectors will have different AOI moving towards shorter wavelengths the spectral band-pass. In Fig. 14 (Right) a narrow band pass in two configurations is shown, the blue in a collimated beam, and in green at a F17 cone. It can be seen that the degradation is very low and has almost no effect in the filter band-pass shape.

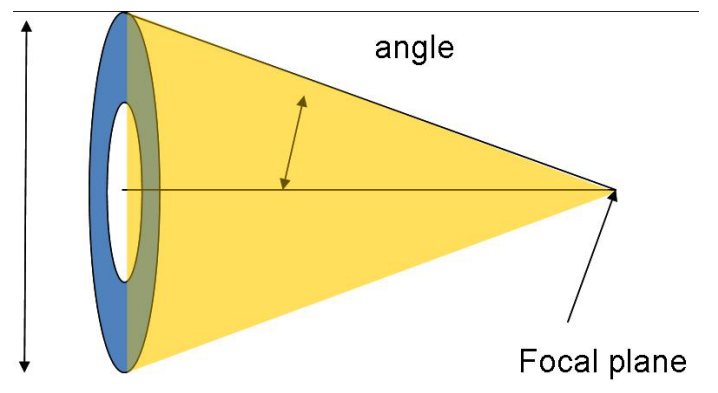

Pupil aperture

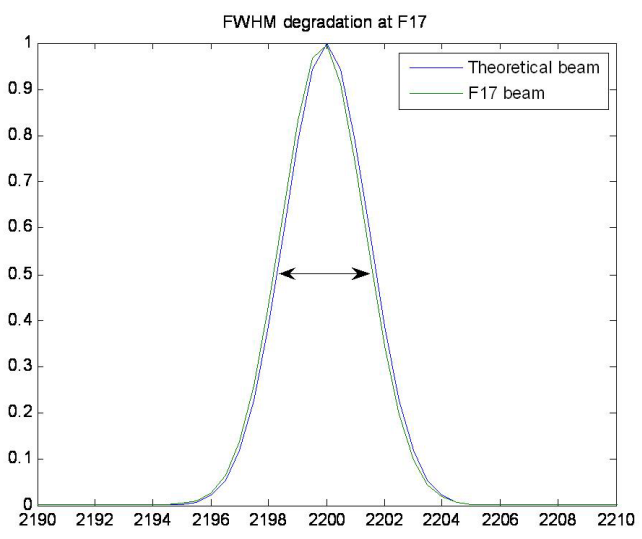

Fig.14. Left - Different angles correspond to circular sectors of the pupil. Right - Comparison of two models with a collimated beam (blue line) and at F17 (green line).

So we can conclude that placing a FP near the GTC F17 focal plane does introduce a minimum degradation in the bandpass and transmission, so that GTC telescope optical design favors the placement of narrow band filters in the entrance telescope focal plane, taking into account scientific performance only. Of course at this entrance focal plane the filters would be very large for the whole CIRCE FOV, what is a big problem in terms of feasibility, risk and cost.

\section{Opto-mechanical design}

The opto-mechanical design has been performed considering the location of the FISIR in the CIRCE optical path should probably be after the filters wheels (see Fig. 15), on the collimated beam. On this position, the collimated beam is spreading very fast, so the diameter of the plates is large. A diameter of $\varnothing 195 \mathrm{~mm}$ should be enough.

Fig. 15 (Right) shows the beam apertures at the first and the last surfaces of the tunable filter. On the first surface the beam aperture diameter is, approximately, $\varnothing 89 \mathrm{~mm}$, while at the exit surface the aperture is $\varnothing 114 \mathrm{~mm}$.

The overall assembly of FISIR is shown in Fig. 16. The "short" piezos that push the first silica plates are shown in color red. These are $12 \mathrm{~mm}$ in length and ø $33 \mathrm{~mm}$ in diameter. The large piezos that support the second plates (in blue) have a length of $33.5 \mathrm{~mm}$, with the same diameter. 
Further analysis should address the possibility of making this diameter a little bit smaller in order to decrease the diameter of the plates. The diameter from the maximum ø $114 \mathrm{~mm}$ free aperture to the maximum size of $\varnothing 195 \mathrm{~mm}$ is necessary to locate the piezos support.

The aluminum mount supports the plates on the CIRCE optical bench. Its width is $250 \mathrm{~mm}$ and its maximum height is $261 \mathrm{~mm}$. It is shown on. Three retainers, $120^{\circ}$ apart, fix the position of the plates with respect to the instrument.
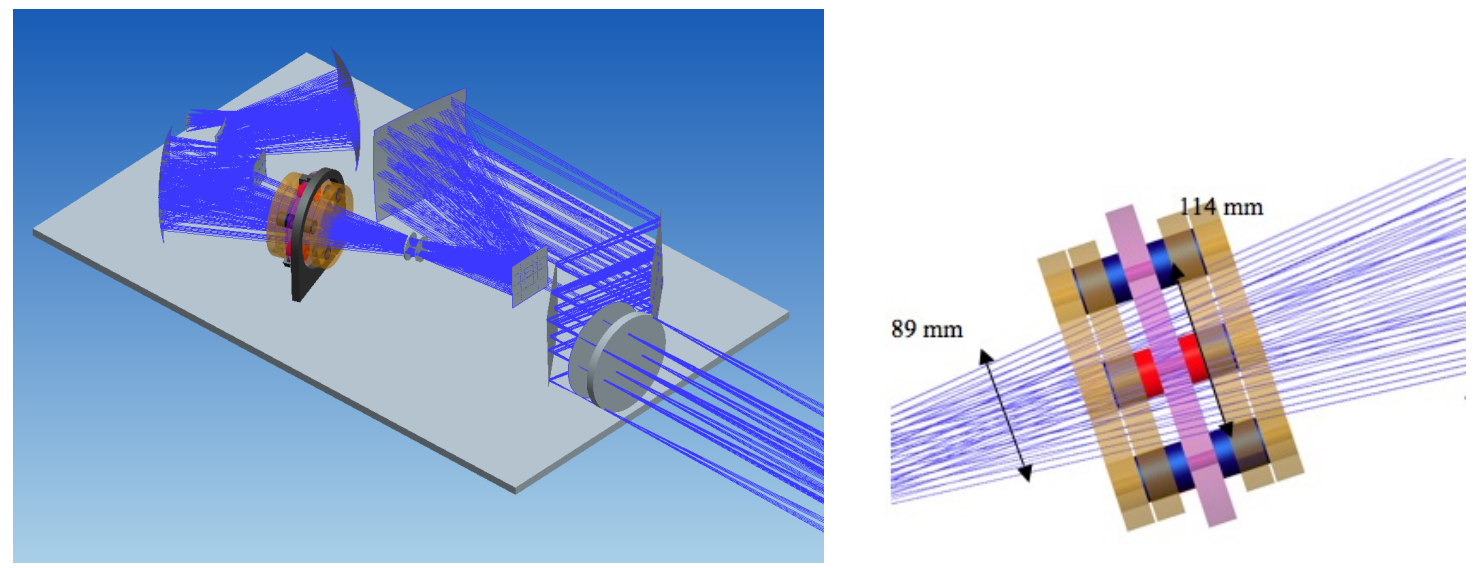

Fig. 15. FISIR in the optical path (Left). Beam apertures through FISIR (Right)
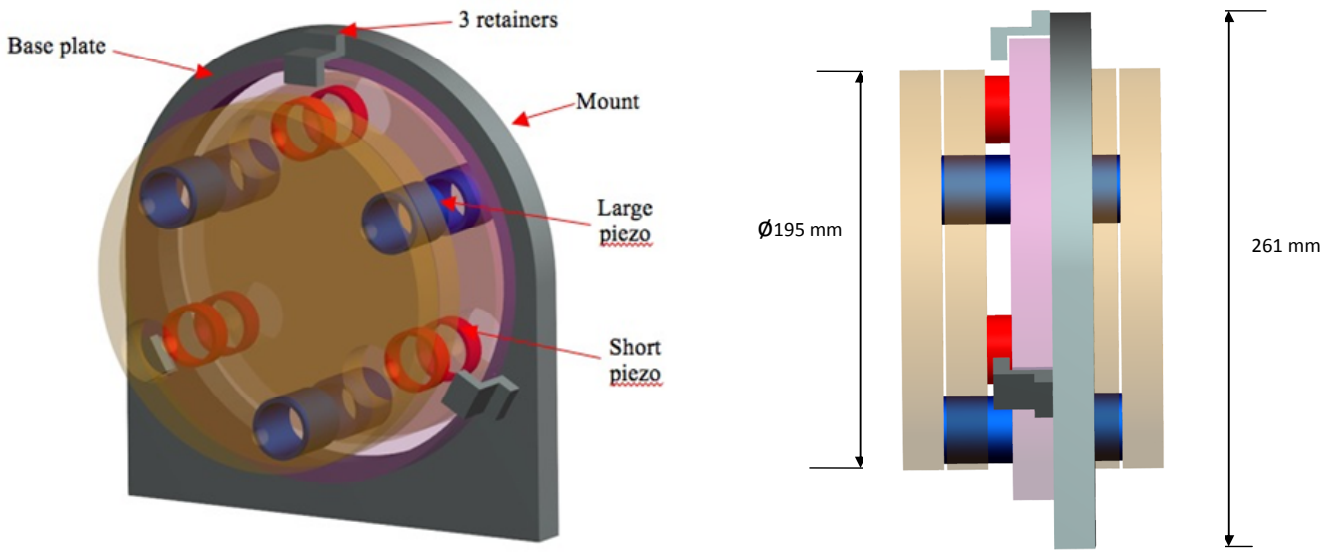

Fig. 16. FISIR Assembly view (Left). Lateral view (Right)

\section{VPH GRATING FOR INFRARED STUDY}

VPHs are currently only used in optical instruments. They could be useful in near-infrared spectrographs if the performance of the VPHs were satisfactory at low temperatures. We performed a study to compile information about the manufacturers, the kind of materials used and the test that should be considered to treat them in cryogenic temperatures.

The study is centered in the following objectives: (1) to analyze the use of Volume Phase Holograms (VPHs) in Infrared wavelengths; (2) to analyze the feasibility of reaching Infrared wavelength range $>1.0 \mu \mathrm{m}$ and (3) to analyze the feasibility of VPHs with temperatures below $200 \mathrm{~K}$.

In order to assess the feasibility of using VPHs at low temperatures, its diffraction efficiency and angular dispersion should be confirmed. Contraction of dichromate gelatin (DCG) with decreasing temperature could cause variations in the line density and profile of diffraction efficiency (the thickness of the gelatin layer is one of the parameters defining diffraction efficiency. 
At this stage we have found few scientist groups that had immersed in the study of VPH at cryogenic temperatures, and all of them have explored near infrared bands not beyond the $2.5 \mathrm{~mm}$. We review the tests performed by different centers $^{3,4}$. All of them repeat basically the same tests based on checking the transmission, the diffraction efficiency and the dispersion and refracted angle response. For that, they perform several heating and cooling cycles from room temperature to cryogenic temperatures that ranges between $200 \mathrm{~K}$ and $100 \mathrm{~K}$.

The instrument configuration was quite similar (a lamp, a monochromator, a vacuum chamber, a camera and/or IR detector). All of them have carried out their tests not beyond the $2.2 \mu \mathrm{m}$ and with cryogenic temperatures over $100 \mathrm{~K}$.

The results are that there is not significant change in the diffraction efficiency in these ranges of temperature (whenever the velocity of the cooling down process is quite slow, of at least 15 hours) and up to $2.2 \mu \mathrm{m}$.

The great majority of them have used for their VPHs BK7 glass and dichromatic gelatin. The transmission of these glasses is over $90 \%$ in the optical and in the range below $2.0 \mu \mathrm{m}$, but there exist other possibilities as the ZnSe crystal for wavelengths well beyond the $2.0 \mu \mathrm{m}$ that could be used to perform further tests beyond this limit.

\section{LICA-UCM SET-UP ACTIVITIES}

This section briefly summarize the main activities performed at the Astrophysics Department of the Universidad Complutense de Madrid (UCM) in its optical laboratory Laboratorio de Instrumentación Científica Avanzada (LICA) in order to improve the laboratory capabilities and have it ready for the optical testing activities now and in the near future.

In particular, all tests required for the characterization of VPH gratings and prisms, the assemblies of gratings with prisms to produce dispersive pupil elements based on VPH technology and to verify the sliced pupil grating prototype (as described in section 2) have been carried out at UCM-LICA. Table 2 summarizes the tests performed for each element.

\begin{tabular}{|c|c|}
\hline Element & Test name \\
\hline VPH-Hologram & VPH Dimensional Verification \\
\hline VPH-Hologram & VPH Surfaces quality \\
\hline VPH-Hologram & VPH Lines/mm \\
\hline VPH-Hologram & VPH PSF (TWFE) \\
\hline VPH-Hologram & VPH Gelatin homogeneity \\
\hline Prisms & Prism Dimensional Verification \\
\hline Prisms & Prism Surface quality \\
\hline Prisms & Prism Wedge Error \\
\hline N/A & Glass edge blackening assembly test \\
\hline N/A & Gel coupling assembly test \\
\hline N/A & Mounted-prisms tilt verification assembly test \\
\hline Assembled unit & System Spectral image quality \\
\hline Assembled unit & System focus and position error \\
\hline Assembled unit & System throughput efficiency \\
\hline
\end{tabular}

Table 2. Tests for characterization of VPH gratings, prisms and the sliced pupil assembly

The set-ups for carrying out these tests were designed and the required components acquired. A CCD and data handling acquisition software were installed for recording the outcome of these tests. Fig. 17 shows some of the set-ups done to perform the tests. We have at laboratory all the means to test and handle elements up to $140 \mathrm{~mm}$. However, MEGARA elements for the GTC will have larger sizes (over $200 \mathrm{~mm}$ ) so that we need to update both our means and our procedure to be able to characterize these elements with enough precision.

Additional preparatory activities are still on-going. In particular, we are upgrading the set-up of a CCD and software for the laboratory in order to improve the capability to perform pupil elements tests in an automatic manner and that can be 
used in future projects. Several manuals are begin produced that contains characterization information to describe the photometric properties of the system. Instructions are also given for use of the test-bench to characterize the CDDs (measure quantum efficiency, filter transmission and CCD flat-fields). In order to follow the study and application of VPHs for infrared we plan to extend this set-up to be able to perform the pupil elements tests at cryogenic temperatures.

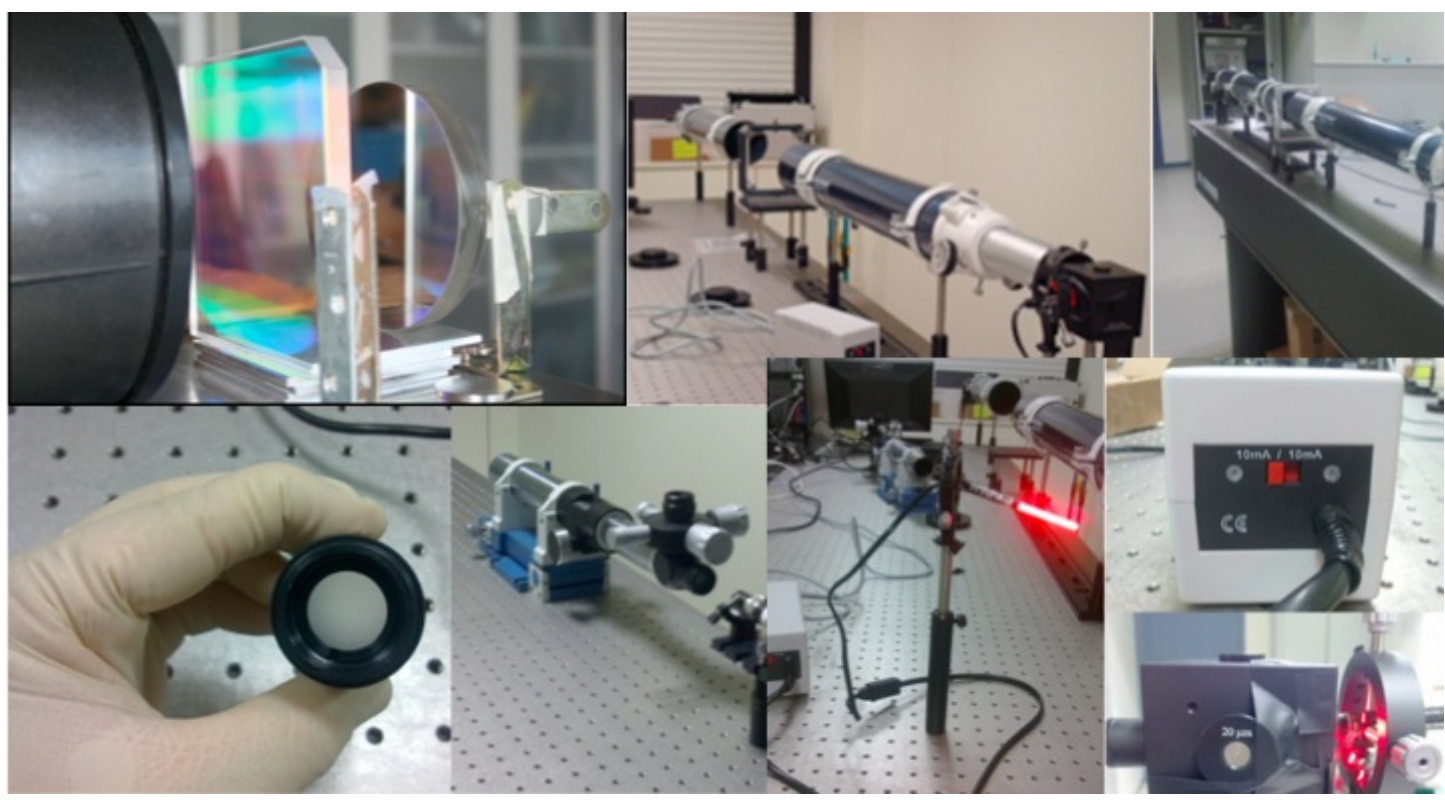

Fig.17. Several images about the set-ups at LICA for the optical tests identified in Table 2.

\section{CONCLUSIONS}

This paper summarizes the findings of the VIENTOS project. Several innovative pupil elements are proposed and studied inside this project. Regarding the sliced pupil grating we present in this paper the system design as well as the test performed for the manufactured prototype. The conclusion from the prototype outcomes is that this system can increase the spectrograph resolution between 3 - 4 times without changing the instrument geometry. We also include the feasibility analysis performed about applying a tunable to CIRCE. Different optical options are discussed that should be reviewed according to the scientific priorities to be defined by the instrument science team. The study done about the current status of using VPHs in Infrared is also summarized as well as a quick overview of the activities done at UCMLICA to prepare the laboratory for testing larger pupil elements in the near-future and in particular for MEGARA project. The VIENTOS project is still on-going. During this last year, we plan to study the feasibility of producing nanotechnology customized filters.

\section{REFERENCES}

[1] Gil de Paz, A, et Al., "MEGARA: the future optical IFU and multi-object spectrograph for the $10.4 \mathrm{~m}$ GTC telescope", Proc. SPIE 8446, pp 182(2012)

[2] Sánchez-Blanco, E.; García-Vargas, M.; Maldonado, M.; Gallego, J.; Gil de Paz, A.; Carrasco, E.; Pérez, A.; Martínez-Delgado, I.; Zamorano, J., "Sliced-pupil grating: a novel concept for increasing spectral resolution", Proc. SPIE Volume 8011, pp. 801(2011)

[3] Ebizuka, Noboru; Ichiyama, Kotaro etal. "Cryogenic VPH grisms for MOIRCS II", Publications of the Astronomical Society of Japan, 63, pp.605-612 (2011)

[4] Arns, J. A.; Smee, S. A.; Barkhouser, R. H.; Benson, M. "Evaluation of VPH gratings at cryogenic temperatures", Proc. SPIE Volume 7018, 70182Q-70182Q-12 (2008). 